\title{
THE TOPOLOGY OF ORDERED ABELIAN GROUPS
}

\section{W. COHEN AND CASPER GOFFMAN}

Introduction. We consider an ordered abelian group $G=[x]$ in which the identity $\theta$ is not isolated: if $x, y>\theta$ in $G$ then $x+y>\theta$; there is an $x \neq \theta$; and if $x>\theta$ there is a $y$ such that $\theta<y<x$. It is found that for each $G$ there is a unique ordinal $\xi^{*}=\xi^{*}(G)$ associated with the topology of $G(\S 1)$.

We study extensions of $G$ which are complete in three different senses. A group is called topologically complete if every fundamental $\xi^{*}$-sequence has a limit. It is called complete in the archimedean sense if it has no proper archimedean extension. It is called order complete if every set bounded above has a least upper bound. These three types will be designated by $t-, a-, o-$ completeness. We show that every ordered abelian group $G$ has a $t$-complete extension $G_{D}$ whose elements are the Dedekindean (\$2) lower segments of $G$ in the sense of Baer [1] $\left(^{1}\right)$. It is pointed out that the $o$-complete extension $G$ consisting of all lower segments of a non-archimedean group $G$ fails to be a group because the non-Dedekindean lower segments do not have inverses $(\S 4)$. The extension $G_{D}$ is isomorphic with the group $G_{C}$ of classes of equivalent fundamental $\xi^{*}$-sequences in $G(\S 2)$. This illuminates the relation between the completion methods of Dedekind and Cantor. It is shown that $a$-completeness implies $t$-completeness but that only a partial converse holds ( $\$ 3$ ).

The Hahn groups $H$ furnish examples of the theory ( $\$ 5)$. It is shown that these groups are $t$-complete and satisfy axioms [1] under which it follows they are of the second $\xi^{*}(H)$-category.

1. Topology and the ordinal $\xi^{*}(G)$. Given an ordinal $\lambda^{*}$ and a single-valued function $x_{\lambda}$ defined on the ordinals $\lambda<\lambda^{*}$ with values in a group $G$, we call $x_{\lambda}$ a $\lambda^{*}$-sequence. A $\lambda^{*}$-sequence is called decreasing if $\lambda_{1}<\lambda_{2}<\lambda^{*}$ implies $x_{\lambda_{1}}>x_{\lambda_{2}}$ in $G$. A $\lambda^{*}$-sequence $x_{\lambda}$ is said to $\lambda^{*}$-converge to $\theta$ if for each $x>\theta$ there is a $\lambda_{x}<\lambda^{*}$ such that $\lambda_{x} \leqq \lambda<\lambda^{*}$ implies $\left|x_{\lambda}\right|<x$ where $|y|=\max [y,-y]$. We write $\lambda^{*}$ - $\lim x_{\lambda}=x$ if $x_{\lambda}-x \lambda^{*}$-converges to $\theta$.

THEOREM 1. The set $\Lambda$ of ordinals $\lambda^{*}$ for which there are decreasing $\lambda^{*}$ sequences $x_{\lambda}$ such that $\lambda^{*}-\lim x_{\lambda}=\theta$ is not empty.

Proof. The nonempty set $P=[x \mid x>\theta]$ can be well-ordered as a $\mu^{*}$-sequence $x_{\mu}$. Let $x_{\mu_{1}}=x_{1}$ and suppose that, for $\nu^{*}<\mu^{*}, x_{\mu_{\nu}}$ has been defined so that, if $\nu<\nu^{*}, \mu_{\nu}$ is the first $\mu$ for which $\tau<\nu$ implies $x_{\mu_{\nu}}<x_{\mu_{\tau}}$. Then $x_{\mu_{\nu}}$ decreases on $\nu<\nu^{*}$. Now either $\nu^{*}-\lim x_{\mu_{\nu}}=\theta$ or there is an $x \in P$ such that $\nu<\nu^{*}$ implies $x \leqq x_{\mu_{\nu}}$. Since $\theta$ is not isolated, there is a first $\mu<\mu^{*}$ such that $\theta<x_{\mu}<x$. Since

Presented to the Society, October 30, 1948; received by the editors September 20, 1948.

(1) Numbers in brackets refer to bibliography at the end of the paper. 
$x_{\mu}<x_{\mu_{\nu}}$ for $\nu<\nu^{*}$ it follows that $\mu>\mu_{\nu}$ for $\nu<\nu^{*}$. We define $\mu_{\nu^{*}}=\mu$. By transfinite induction it follows that there is a $\lambda^{*}$-sequence $x_{\mu_{\nu}}, \nu<\lambda^{*}$, such that $\lambda^{*}-\lim x_{\mu_{\nu}}=\theta$. Hence $\lambda^{*} \in \Lambda$.

The nonempty set $\Lambda=\left[\lambda^{*}\right]$ has a first element which we denote by $\xi^{*}=\xi^{*}(G)$. There is a decreasing $\xi^{*}$-sequence $a_{\xi}$ in $G$ such that $\xi^{*}$-lim $a_{\xi}=\theta$ by Theorem 1 . We shall make extensive use of the sequence $a_{\xi}$ in the sequel.

Theorem 2. The ordinal $\xi^{*}(G)$ is a limit number such that if $\eta^{*}<\xi^{*}$ and $\xi_{\eta}$ is a single-valued function on $\eta<\eta^{*}$ to $\xi<\xi^{*}$, then $\sup \left[\xi_{\eta} \mid \eta<\eta^{*}\right]<\xi^{*}\left({ }^{2}\right)$.

Proof. $\xi^{*}=\xi^{*}(G)$ is a limit number. Otherwise $\xi^{*}=\eta+1$ and $\theta<a_{\eta} \leqq a_{\xi}$ for $\xi<\xi^{*}$ which is contrary to $\xi^{*}-\lim a_{\xi}=\theta$. Now suppose there are $\xi^{*}<\xi^{*}$ and a function $\xi_{\zeta}$ on $\zeta<\zeta^{*}$ to $\xi<\xi^{*}$ such that $\sup \left[\xi_{\zeta} \mid \zeta<\zeta^{*}\right]=\xi^{*}$. It may be assumed without loss of generality that $\zeta_{1}<\zeta_{2}<\zeta^{*}$ implies $\xi_{\zeta_{1}} \leqq \xi_{\zeta_{2}}$. We show that $\zeta^{*}-\lim a_{\xi_{\zeta}}=\theta$. If $x>\theta$ in $G$ there is $\eta<\xi^{*}$ such that $\theta<a_{\eta}<x$. There is $\zeta(\eta)<\zeta^{*}$ such that $\eta<\xi_{\zeta(\eta)}<\xi^{*}$. Since $a_{\xi}$ decreases on $\xi<\xi^{*}, \theta<a_{\xi_{\xi}}<a_{\xi_{\zeta(\eta)}}<a_{\eta}<x$ if $\zeta(\eta)<\zeta<\xi^{*}$ and so $\zeta^{*}$-lim $a_{\xi_{\xi}}=\theta$. Hence $\zeta^{*} \in \Lambda$ and so $\xi^{*} \leqq \zeta^{*}$ since $\xi^{*}=\xi^{*}(G)$ is the first element of $\Lambda$. This contradicts the assumption $\zeta^{*}<\xi^{*}$.

A neighborhood system $V=\left[U_{\xi}(x)\right]$ for $G$ is defined as follows:

$$
\begin{array}{rlr}
U_{\xi} & =U_{\xi}(\theta)=\left[x|| x \mid<a_{\xi}\right], & \xi<\xi^{*}, \\
U_{\xi}(x) & =x+U_{\xi}=\left[x+y \mid y \in U_{\xi}\right] .
\end{array}
$$

We shall refer to $\xi^{*}$-sequences hereafter simply as sequences. Before stating the basic properties of the neighborhood system $U$ we prove three lemmas.

Lemma 1. For each positive integer $n, \xi^{*}-\lim n a_{\xi}=\theta$.

Proof. Suppose $n=2$. If $x>\theta$ there is a $\xi_{1}<\xi^{*}$ such that $\theta<a_{\xi_{1}}<x$. There is a $\xi_{2}<\xi^{*}$ such that $\theta<a_{\xi_{2}}<x-a_{\xi_{1}}$. If $\max \left[\xi_{1}, \xi_{2}\right] \leqq \xi<\xi^{*}$, then $\theta<a_{\xi}+a_{\xi}<a_{\xi_{1}}$ $+a_{\xi_{2}}<x$ and so $\xi^{*}-\lim 2 a_{\xi}=\theta$. Induction completes the proof.

Lemma 2. For each positive integer $n$ and $\eta<\xi^{*}$ there is a $\xi=\xi(n, \eta)<\xi^{*}$ such that $n U_{\xi}=\left[x_{1}+\cdots+x_{n} \mid x_{i} \in U_{\xi}\right] \subset U_{\eta}$.

Proof. Suppose $n=2$. There is a $\xi=\xi(2, \eta)$ such that $\theta<a_{\xi}+a_{\xi}<a_{\eta}$ by Lemma 1. If $x_{1}, x_{2} \in U_{\xi}$, then $\left|x_{1}+x_{2}\right| \leqq\left|x_{1}\right|+\left|x_{2}\right|<2 a_{\xi}<a_{\eta}$. Hence $2 U_{\xi} \subset U_{\eta}$. Induction completes the proof.

LEMMA 3. $-U_{\xi}=\left[-x \mid x \in U_{\xi}\right]=U_{\xi}$.

Proof. The proof is clear.

TheOREM 3. The neighborhood system $U$ has the properties:

$\alpha . \bigcap_{\xi<\xi^{*}} U_{\xi}(x)=x$.

B. $\xi_{1}<\xi_{2}<\xi^{*}$ implies $U_{\xi_{1}}(x) \supset U_{\xi_{2}}(x)$.

(2) $\xi^{*}(G)$ has property $\left(^{*}\right)$ of $[1]$. 
$\gamma . \eta<\xi^{*}$ implies there is $\xi(\eta)$ such that $\eta<\xi(\eta)<\xi^{*}$ and if $U_{\xi(\eta)}(y) \cap U_{\xi(\eta)}(x)$ $\neq 0$ then $U_{\xi(\eta)}(y) \subset U_{\eta}(x)$.

ס. $\eta^{*}<\xi^{*}$ implies $\bigcap_{\xi<\eta^{*}} U_{\xi}(x)$ is open $\left({ }^{3}\right)$.

Proof. Property $\alpha$ follows from $\xi^{*}-\lim a_{\xi}=\theta$. Property $\beta$ follows from the decreasing character of the $a_{\xi}$. For property $\gamma$ : given $\eta<\xi^{*}$ there is a $\xi(\eta)$ for which $3 U_{\xi(\eta)} \subset U_{\eta}$ by Lemma 2 . By property $\beta$ we may choose $\xi(\eta)>\eta$. Now if $z \in U_{\xi(\eta)}(y) \cap U_{\xi(\eta)}(x)$ and $w \in U_{\xi(\eta)}(y)$, then $z-y, z-x, w-y \in U_{\xi(\eta)}$. Using Lemma 3 we have

$$
w-x=(w-y)+(y-z)+(z-x) \in 3 U_{\xi(\eta)} \subset U_{\eta} .
$$

Hence $w \in U_{\eta}(x)$ and so $U_{\xi(\eta)}(y) \subset U_{\eta}(x)$. For property $\delta$ : Suppose $y$ $\in \bigcap_{\xi<\eta^{*}} U_{\xi}(x)$. We have $y-x \in \bigcap_{\xi<\eta^{*}} U_{\xi}$ and so $|y-x|<a_{\xi}$ for $\xi<\eta^{*}$. Hence $b_{\xi}=a_{\xi}-|y-x|>\theta$ and decreases on $\xi<\eta^{*}$. Because of the minimal character of $\xi^{*} \in \Lambda$ (Theorem 1), $\eta^{*}-\lim b_{\xi} \neq \theta$ and there is an $a_{\zeta}<b_{\xi}$ for $\xi<\eta^{*}$. Now if $|z-y|<a_{\zeta}$, then

$$
|z-x| \leqq|z-y|+|y-x|<a_{\zeta}+|y-x|<b_{\xi}+|y-x|=a_{\xi}
$$

for all $\xi<\eta^{*}$. Hence $U_{\zeta}(y) \subset \bigcap_{\xi<\eta^{*}} U_{\xi}(x)$.

THEOREM 4. $G$ is a topological group relative to the neighborhood system $U$.

Proof. Consider $x_{0}, y_{0} \in G$ and $U_{\eta}\left(x_{0}+y_{0}\right) \in \mathcal{V}$. By Lemma 2 there is a $U_{\xi_{\eta}}$ such that $2 U_{\xi_{\eta}} \subset U_{\eta}$. Now if $x \in U_{\xi_{\eta}}\left(x_{0}\right), y \in U_{\xi_{\eta}}\left(y_{0}\right)$, then $\left(x-x_{0}\right)+\left(y-y_{0}\right)$ $\in U_{\xi_{\eta}}+U_{\xi_{\eta}} \subset U_{\eta}$. Hence $x+y \in U_{\eta}\left(x_{0}+y_{0}\right)$ and $x+y$ is a continuous function of its two variables.

2. Lower segments and topological completeness. By a lower segment in $G$ is meant a nonempty, proper subset $X$ of $G$ such that if $x \in X$, then all $y<x$ and some $z>x$ are in $X$. For each $x_{0} \in G$ the set $X\left(x_{0}\right)=\left[x \mid x<x_{0}\right]$ is a lower segment in $G$. A lower segment is called Dedekindean if $y>\theta$ in $G$ implies there is an $x \in X$ such that $x+y \notin X$. This concept was introduced by $\mathrm{R}$. Baer [1] in finding necessary and sufficient conditions for absolute closure. It is well known that the set of lower segments of a non-archimedean group is not a group. However, the Dedekindean lower segments form a group $G_{D}$.

A sequence $x_{\xi}$ in $G$ is called fundamental if for every $\eta<\xi^{*}$ there are $\xi_{\eta}<\xi^{*}$ and $y_{\eta} \in G$ such that $\xi_{\eta} \leqq \xi<\xi^{*}$ implies $x_{\xi} \in U_{\eta}\left(y_{\eta}\right)$. It is known [2] that $x_{\xi}$ is a fundamental sequence if and only if $x_{\xi} \in U_{\eta}\left(x_{\xi_{\eta}}\right)$ for $\xi_{\eta} \leqq \xi<\xi^{*}$ and all $\eta<\xi^{*}$. We shall write $\lim x_{\xi}=x$ if for $\eta<\xi^{*}$ there is $\xi_{\eta}$ such that $x_{\xi} \in U_{\eta}(x)$ if $\xi_{\eta} \leqq \xi<\xi^{*}$. The group $G$ is called $t$-complete if every fundamental sequence has a limit in $G$. It is shown that $G_{D}$ is $t$-complete.

Theorem 5. $X$ is a Dedekindean lower segment if and only if there is a fundamental sequence $x_{\xi}$ cofinal with $X$ such that $x_{\xi}+a_{\xi} \notin X$.

(3) Properties $\alpha, \beta, \gamma$ are axioms 1, 2, 3 in [1]. The space of [1] satisfies axiom 4 which is stronger than $\delta$. The Hahn groups satisfy $\alpha, \beta, \gamma$ and 4 (Theorem 14 , below). 
Proof. Suppose $X$ is a Dedekindean lower segment. Then for each $\xi<\xi^{*}$ there is an $x_{\xi} \in X$ such that $x_{\xi}+a_{\xi} \notin X$. Now if $x \in X$ there is in $X$ a $z>x$. There is a $\xi<\xi^{*}$ such that $\theta<a_{\xi}<z-x$. Since $z \in X$ and $x_{\xi}+a_{\xi} \notin X, z<x_{\xi}$ $+a_{\xi}<x_{\xi}+z-x$ and so $x<x_{\xi}$. Hence the sequence $x_{\xi}$ is cofinal with $X$.

We show that $x_{\xi}$ is a fundamental sequence. If $\eta<\xi<\xi^{*}$, then $x_{\eta}-a_{\eta}<x_{\xi}$. Otherwise for some $\eta<\xi<\xi^{*}, x_{\xi} \leqq x_{\eta}-a_{\eta}$. Since $\eta<\xi$ implies $\theta<a_{\xi}<a_{\eta}$, $x_{\xi}+a_{\xi} \leqq x_{\eta}-a_{\eta}+a_{\xi}<x_{\eta} \in X$ and so $x_{\xi}+a_{\xi} \in X$ which is false. On the other hand, since $x_{\xi} \in X$ and $x_{\eta}+a_{\eta} \notin X$ for all $\xi, \eta<\xi^{*}, x_{\xi}<x_{\eta}+a_{\eta}$. Hence $x_{\xi} \in U_{\eta}\left(x_{\eta}\right)$ for $\eta \leqq \xi<\xi^{*}$ and $x_{\xi}$ is a fundamental sequence.

Now suppose that $x_{\xi} \in X$ and $x_{\xi}+a_{\xi} \notin X$ for $\xi<\xi^{*}$. Then for $y>\theta$ there is an $a_{\xi}$ such that $\theta<a_{\xi}<y$ and so $x_{\xi}+y \notin X$ since $x_{\xi}+y>x_{\xi}+a_{\xi} \notin X$. Hence $X$ is Dedekindean.

The lower segments $X\left(x_{0}\right)$ are Dedekindean since $x_{0}-a_{\xi}$ is a fundamental sequence cofinal with $X\left(x_{0}\right)$ such that $x_{0}=\left(x_{0}-a_{\xi}\right)+a_{\xi} \notin X\left(x_{0}\right)$. If we define $X\left(x_{1}+x_{2}\right)=X\left(x_{1}\right)+X\left(x_{2}\right)$ and $X\left(x_{1}\right)>X\left(x_{2}\right)$ if $x_{1}>x_{2}$, it is clear that the set of lower segments $X(x), x \in G$, form an abelian group isomorphic with $G$. We denote by $G_{D}$ the set of Dedekindean lower segments. For $X_{1}, X_{2} \in G_{D}$ we define

$$
\begin{aligned}
& X_{1}+X_{2}=\left[x_{1}+x_{2} \mid x_{i} \in X_{i}, i=1,2\right], \\
& X_{1}>X_{2} \text { if } X_{1} \supset X_{2} \text { properly. }
\end{aligned}
$$

Lemma 4. $X_{1}, X_{2} \in G_{D}$ implies $X_{1}+X_{2} \in G_{D}$.

Proof. Since $X_{i} \neq 0, i=1,2, X_{1}+X_{2} \neq 0$. Since $X_{i} \neq G$ there are $y_{i} \notin X_{i}$. Now $y_{1}+y_{2} \notin X_{1}+X_{2}$. Otherwise $y_{1}+y_{2}=x_{1}+x_{2}, x_{i} \in X_{i}$. Then $y_{1}+\left(y_{2}-x_{2}\right)$ $=x_{1} \in X_{1}$ and, since $y_{2} \in X_{2}, x_{2} \in X_{2}$, we have $y_{2}-x_{2}>\theta$ so that $y_{1}<x_{1}, y_{1} \in X_{1}$ which is false. Thus $X_{1}+X_{2}$ is a proper subset of $G$. Consider $x=x_{1}+x_{2} \in X_{1}$ $+X_{2}, x_{i} \in X_{i}$. If $y<x_{1}+x_{2}$ then $y-x_{2}<x_{1}, y-x_{2} \in X_{1}$, and $y=\left(y-x_{2}\right)+x_{2}$ $\in X_{1}+X_{2}$. There is a $z_{1} \in X_{1}$ such that $x-x_{2}=x_{1}<z_{1}$. Then $x<z_{1}+x_{2} \in X_{1}$ $+X_{2}$. Hence $X_{1}+X_{2}$ is a lower segment in $G$. We show that $X_{1}+X_{2}$ is Dedekindean. Since each $X_{i}$ is Dedekindean there is a sequence $x_{i \xi} \in X_{i}$ such that $x_{i \xi}+a_{\xi} \notin X_{i}$. Then $x_{1 \xi}+x_{2 \xi}+2 a_{\xi} \notin X_{1}+X_{2}$. Now for $x>\theta$ there is an $\eta<\xi^{*}$ such that $2 a_{\eta}<x$. Hence $x_{1 \eta}+x_{2 \eta}+x>x_{1 \eta}+x_{2 \eta}+2 a_{\eta}$ so that $x_{1 \eta}+x_{2 \eta}$ $\in X_{1}+X_{2}$ while $x_{1 \eta}+x_{2 \eta}+x \notin X_{1}+X_{2}$. Hence $X_{1}+X_{2}$ is Dedekindean.

Lemma 5. $X_{1}+X_{2}=X_{2}+X_{1} ; X_{1}+\left(X_{2}+X_{3}\right)=\left(X_{1}+X_{2}\right)+X_{3}$ if $X_{i} \in G_{D}$.

Proof. The proof is clear.

Lemma 6. $X \in G_{D}$ implies $X(\theta)+X=X$.

Proof. If $x \in X$ and $y \in X(\theta)$, then $x+y<x$ and so $X+X(\theta) \subset X$. If $x \in X$ there is a $z \in X$ such that $x<z$. Then $x-z \in X(\theta)$ and $x=z+(x-z) \in X(\theta)+X$. Hence $X \subset X+X(\theta)$. We shall denote $X(\theta)$ by $\Theta$.

LemmA 7. If $X \in G_{D}, Y=[y \mid-y \notin X], Z=Y-[\sup Y]$ where the set [sup $Y$ ] may be empty, then $Z \in G_{D}$ and $X+Z=\Theta$. 
Proof. Since $Y$ contains more than one element and is not $G, Z \neq 0, G$. Consider $z \in Z$. If $w<z$, then $-z<-w$ and, since $-z \notin X,-w \notin X, w \in Z$. Further since $z<\sup Y$ or sup $Y$ does not exist, there is $w \in Z$ such that $z<w$. Hence $Z$ is a lower segment.

We show that $Z$ is Dedekindean. Consider $w>\theta$ and $a_{\xi}<w$. Since $X$ is Dedekindean there is an $x \in X$ such that $x+a_{\xi} \notin X$. Now $-\left(x+a_{\xi}\right) \in Y$ and, since $x+a_{\xi}<x+w,-(x+w)<-\left(x+a_{\xi}\right)$. Hence $-(x+w) \neq \sup \quad Y$ and $x+w \notin X$. Therefore $-(x+w) \in Y-[\sup Y]=Z$. Now $-x=-(x+w)+w$ is not in $Z$. Otherwise $-x \in Z \subset Y$ and $x \notin X$ which is false. Thus for any $w>\theta$ there is $z=-(x+w) \in Z$ such that $z+w=-(x+w)+w=-x \notin Z$ and $Z$ is Dedekindean.

Finally we show that $X+Z=\Theta$. Consider any $u<\theta$ and $a_{\xi}<-u$. Since $X$ is Dedekindean there is an $x \in X$ such that $x+a_{\xi} \notin X$. Hence $-x-a_{\xi} \in Y$ and, since $u-x<-x-a_{\xi}, u-x \in Z$. Thus $u=(u-x)+x \in Z+X$ and $\Theta \subset Z+X$. On the other hand if $x \in X$ and $z \in Z$, then, since $-z \notin X, x<-z$. Hence $x+z<\theta, x+z \in \Theta$ and $X+Z \subset \Theta$, which completes the proof.

Theorem 6. The set $G_{D}$ of Dedekindean lower segments in $G$ is an ordered abelian extension of $G$.

Proof. Lemmas $4, \cdots, 7$ show that $G_{D}$ is an ordered abelian group. The set of $X(x), x \in G$, is a subgroup of $G_{D}$ isomorphic with $G$.

In discussing the group $G_{D}$ we shall denote $X(x) \in G_{D}$ by $x$, for example, $X+y=X+X(y)$ and $X>X(y)$ may be written $X>y$. The group $G_{D}$ has the associated minimal ordinal (Theorem 1) $\xi^{*}\left(G_{D}\right)$.

TheOREM 7. $\xi^{*}\left(G_{D}\right)=\xi^{*}(G)$.

Proof. First we show that $\xi^{*}\left(G_{D}\right) \leqq \xi^{*}(G)$. We note that $X\left(a_{\xi}\right), \xi<\xi^{*}$, decrease and are positive in $G_{D}$. Consider $X>\Theta$. There is an $x \in X$ such that $x>\theta$. There is a $\xi<\xi^{*}$ such that $\theta<a_{\xi}<x$. Hence $\Theta<X\left(a_{\xi}\right)<X(x)<X$. Since the $X\left(a_{\xi}\right)$ decrease, $\xi^{*}-\lim X\left(a_{\xi}\right)=\Theta$ and so $\xi^{*}\left(G_{D}\right) \leqq \xi^{*}(G)$. Now let $\eta^{*}=\xi^{*}\left(G_{D}\right)$. Then there is a decreasing $\eta^{*}$-sequence $X_{\eta}$ in $G_{D}$ such that $\eta^{*}-\lim X_{\eta}=\Theta$. Since the $X_{\eta}$ are positive lower segments in $G$ there is, for each $\eta<\eta^{*}$, an $x_{\eta} \in X_{\eta}$ such that $\theta<x_{\eta}$. For any $a_{\xi} \in G, X\left(a_{\xi}\right)>\Theta$ and there is an $\eta_{\xi}<\eta^{*}$ such that if $\eta_{\xi} \leqq \eta<\eta^{*}$, then $\Theta<X_{\eta}<X\left(a_{\xi}\right)$. Now $x_{\eta} \in X_{\eta} \subset X\left(a_{\xi}\right)$ and so $\theta<x_{\eta}<a_{\xi}$ for $\eta_{\xi} \leqq \eta<\eta^{*}$. Hence $\eta^{*}$-lim $x_{\eta}=\theta$. By transfinite induction and Theorem 2 a decreasing $\zeta^{*}$-subsequence $\zeta^{*} \leqq \eta^{*}$ can be chosen from $x_{\eta}$. It follows that $\xi^{*}(G) \leqq \zeta^{*} \leqq \xi^{*}\left(G_{D}\right)$ and the proof is complete.

Corollary. The system $V_{D}=\left[U_{\xi}(X)\right]$ where

$$
U_{\xi}(X)=\left[Y|| Y-X \mid<a_{\xi}\right], \quad \xi<\xi^{*},
$$

is a neighborhood system for $G_{D}$ having the properties of Theorem 3 . 
Theorem 8. $G_{D}$ is t-complete.

Proof. Let $X_{\xi}$ be a fundamental sequence in $G_{D}$. Consider $Y_{\eta}=\bigcup_{\eta \leqq \xi<\xi^{*}} X_{\xi}$, $Y=\bigcap_{\eta}<\xi^{*} Y_{\eta}, X=Y-[\sup Y]$. Since $X_{\xi}$ is a fundamental sequence, for each $\eta<\xi^{*}$ there is a $\xi_{\eta}>\eta$ such that $X_{\xi_{\eta}}-a_{\eta}<X_{\xi}<X_{\xi_{\eta}}+a_{\eta}$ if $\xi_{\eta} \leqq \xi<\xi^{*}$. Hence

$$
Y=\bigcap_{\zeta<\xi^{*}} Y_{\zeta} \cap Y_{\xi_{\eta}}=\bigcup_{\xi_{\eta} \leqq \xi<\xi^{*}} X_{\xi} \subset X_{\xi_{\eta}}+a_{\eta}, \quad \eta<\xi^{*},
$$

and so $X \subset Y \subset X_{\xi_{\eta}}+a_{\eta} \neq G$. For every $\eta, \quad \zeta<\xi^{*}, Y_{\xi} \supset X_{\xi} \supset X_{\xi_{\eta}}-a_{\eta}$ if $\xi$ $>\max \left[\zeta, \xi_{\eta}\right]$. Hence

$$
Y=\bigcap_{\zeta<\xi^{*}} Y_{\zeta} \supset X_{\xi_{\eta}}-a_{\eta}, \quad \eta<\xi^{*},
$$

and so $X=Y-[\sup Y] \neq 0$.

If $x \in X$, then $x \in Y_{\eta}, \eta<\xi^{*}$, and $x \in X_{\xi_{x, \eta}}$ for some $\xi_{x, \eta} \geqq \eta$. Now $y<x$ and $X_{\xi_{x, \eta}} \in G_{D}$ imply $y \in X_{\xi_{x, \eta}} \subset Y_{\eta}, \eta<\xi^{*}$. Hence $y \in Y$. But $y<x \in Y$ and so $y \neq \sup Y$ and $y \in X$. Since $X=Y-[\sup Y]$ and $G$ is not discrete, $x \in X$ implies there is a $w \in X$ such that $x<w$. Thus $X$ is a lower segment in $G$.

We show that $X \in G_{D}$. Consider $x>\theta$ and $\zeta_{0}<\xi^{*}$ such that $3 a_{\zeta}<x$ for $\zeta_{0} \leqq \zeta<\xi^{*}$. Now from (1), (2) we have

$$
X_{\xi_{\zeta}}-a_{\zeta} \subset Y \subset X_{\xi_{\xi}}+a_{\zeta}, \quad \zeta_{0} \leqq \zeta<\xi^{*} .
$$

Since $X_{\xi_{\zeta_{0}}}-a_{\zeta_{0}} \in G_{D}$ there is $y \in X_{\xi_{\zeta_{0}}}-a_{\zeta_{0}}$ such that $y+a_{\zeta_{0}} \notin X_{\xi_{\zeta_{0}}}-a_{\zeta_{0}}$. Then $y+x>y+3 a_{\xi_{0}} \notin X_{\xi_{50}}+a_{\zeta_{0}}$. Hence $y+x \notin X \subset Y \subset X_{\xi_{\zeta_{0}}}+a_{\zeta_{0}}$. Since $X_{\xi_{\zeta_{0}}}-a_{\zeta_{0}}$ has no maximum and is contained in $Y, y \neq \sup Y$. Thus $y \in X_{\xi_{0}}-a_{\xi_{0}} \subset Y$ $-[\sup Y]=X$ and $y+x \notin X$ and $X$ is a Dedekindean lower segment. (3)

We show that $\lim X_{\xi}=X$. Since $X=Y-[\sup Y]$ and $a_{\zeta}>\theta$ we have from

$$
\begin{aligned}
& X_{\xi_{\zeta}}-2 a_{\zeta}<X<X_{\xi_{\zeta}}+2 a_{\zeta}, \\
& \left|X-X_{\xi_{\zeta}}\right|<2 a_{\zeta},
\end{aligned}
$$

For $\eta<\xi^{*}, 2 a_{\zeta}<a_{\eta}$ for $\zeta_{\eta} \leqq \zeta<\xi^{*}$ by Lemma 1 . Hence $\left|X_{\xi_{\zeta}}-X\right|<a_{\eta}$ for $\max \left[\zeta_{0}, \zeta_{\eta}\right] \leqq \zeta<\xi^{*}$ and so $\lim X_{\xi_{\xi}}=X$. Since $X_{\xi_{\xi}}$ is a subsequence of the fundamental sequence $X_{\xi}, \lim X_{\xi}=X$.

The facts that the group $G_{D}$ consists of the Dedekindean lower segments in $G$ and that the set of all lower segments in $G$ do not in general form a group [3] together with the appearance of fundamental sequences in Theorem 5 and the proof of Theorem 8 cast light on the roles played by the Dedekind cut and the Cantor fundamental sequence in completeness theory. There is an equivalence between the Dedekindean lower segments and the classes of equivalent fundamental sequences. Two fundamental sequences $x_{\xi}, y_{\xi}$ in $G$ are equivalent if $\lim x_{\xi}-y_{\xi}=\theta$. It is clear that this definition separates the set of all fundamental sequences in $G$ into disjoint equivalence classes. These classes form an ordered abelian group $G_{C}$ isomorphic with $G_{D}$. The proof of 
this, which follows classical lines, can be supplied by the reader.

3. Completeness in the archimedean sense. Completeness in the sense of Archimedes is a concept introduced by Hilbert [4] in the foundations of geometry. Two elements $x, y>\theta$ in an ordered abelian group $G$ are called relatively archimedean if there are positive integers $n, m$ such that $n x \geqq y$ and $m y \geqq x$ in $G$. If every pair $x, y>\theta$ in $G$ are relatively archimedean, then $G$ is an archimedean group. An extension $H$ of $G$ is called an archimedean extension if for every $y \in H, y>\theta_{H}$, there is an $x \in G$ such that $x, y$ are relatively archimedean in $H$. A group $G$ is called $a$-complete if $G$ has no proper archimedean extension. We indicate the relation between $t$ - and $a$-completeness.

Theorem 9. If $G$ is a-complete, then $G$ is t-complete.

Proof. Suppose $G$ is not $t$-complete. Then $G_{D}$, being a $t$-complete extension (Theorem 8), is a proper extension of $G$. We show that $G_{D}$ is an archimedean extension of $G$. Consider $X>\Theta$ in $G_{D}$. There is a fundamental sequence $x_{\xi}$ cofinal with $X$ such that $x_{\xi}+a_{\xi} \notin X$ (Theorem 5). There are $x>\theta$ in $G$ and $\eta<\xi^{*}$ such that for $\eta<\xi<\xi^{*}, x<x_{\xi}$. There is a $\zeta>\eta$ such that for $\zeta \leqq \xi<\xi^{*}$, $\left|x_{\xi}-x_{\zeta}\right|<x$. Now $x_{\zeta} \in X$ and $x_{\zeta}+x_{\zeta}>x_{\zeta}+x>x_{\xi}$ if $\zeta \leqq \xi<\xi^{*}$. Hence $x_{\zeta}+x_{\zeta} \geqq X$ and so $G_{D}$ is an archimedean as well as a proper extension of $G$. This contradicts the hypothesis and the theorem follows.

The converse of this theorem is false as the following example shows. Let $G$ be the set $[g]$ of integer valued functions $g(n)$ on the set of positive integers and let $H$ be the set [h] of real functions $h(n)$ on the same domain. If $f_{1}, f_{2}$ are functions in either set we define $f_{1}<f_{2}$ if $f_{1}(m)<f_{2}(m)$ where $m$ is the first $n$ for which at least one of the functions does not vanish. With the usual definition of addition and the null function, $G$ is an ordered abelian group and $H$ is a proper extension of $G$. We define the functions $a_{m}=a_{m}(n)=1,0$ according as whether $n=m, \neq m$. In both $G$ and $H, \omega-\lim a_{m}=\theta$. If we denote by $f$ an element either of $G$ or of $H$, the neighborhoods $U_{k}(f)=\left[f^{\prime}|| f^{\prime}-f \mid<a_{k}\right]$ are equivalent to the sets $V_{k}(f)=\left[f^{\prime} \mid f^{\prime}(n)=f(n), n \leqq k\right]$. $G$ is $t$-complete. For $g_{\nu}$ is a fundamental sequence in $G$ if and only if for each $k$ there is a $\nu(k)$ such that, for $\nu \geqq \nu(k)$ and $n \leqq k, g_{\nu}(n)=g_{\nu(k)}(n)$. The function $g=g_{\nu(n)}(n)$ is the limit of $g_{\nu} . H$ is an archimedean extension of $G$. For consider $h=h(n)>\theta$. There is a first $n=m$ for which $h(n) \neq 0$. Then $h(m)>0$ since $h>\theta$. The function $a_{m} \in G$ is such that, for some integer $k, k h(m)>1=a_{m}(m)$ and $k a_{m}(m)>h(m)$. Therefore the $t$-complete group $G$, having $H$ as a proper archimedean extension, is not $a$-complete.

A partial converse of the previous theorem is:

THEOREM 10. If $G$ is t-complete and is dense in every archimedean extension, then $G$ is a-complete.

Proof. Let $H$ be an archimedean extension of $G$. The sequence $a_{\xi}$ which decreases to $\theta$ in $G$ decreases to $\theta$ in $H$. Otherwise there is $y>\theta$ in $H$ such that 
there is no $x \in G$ between $\theta$ and $y$ and $G$ is not dense in $H$. Now for $z \in H$ and $\xi<\xi^{*}$ there is an $x_{\xi} \in G$ such that $z-a_{\xi}<x_{\xi}<z+a_{\xi}$ since $G$ is dense in $H$. From the decreasing character of the $a_{\xi}$ with the limit $\theta$ in $G$ it follows that $x_{\xi}$ is a fundamental sequence. Since $G$ is $t$-complete, $\lim x_{\xi}=z \in G$. Hence $H=G$ and $G$ is $a$-complete.

4. Order completion. The set $\bar{G}$ of all lower segments in $G$ is not a group in general. We note that this failure is due to the existence of nonDedekindean lower segments since such segments have no inverses. If $G$ is a non-archimedean group there are non-Dedekindean lower segments in $\bar{G}$. For: There are $x, y \in G$ such that $\theta<n x<y$ for all positive integers $n$. The lower segment $X=\left[z \mid z<n_{z} x\right]$ is non-Dedekindean.

THEOREM 11. If $\xi^{*}=\xi^{*}(G)>\omega$, then $G$ is non-archimedean.

Proof. There is a $\xi_{1}<\xi^{*}$ such that $2 a_{\xi_{1}}<a_{1}$ (Lemma 1) and for $\xi_{n}$ there is $\xi_{n+1}<\xi^{*}$ such that $2 a_{\xi_{n+1}}<a_{\xi_{n}}, n=1,2, \cdots$. Now by Theorem 2, since $\xi^{*}>\omega$, there is a $\zeta$ such that sup $\left[\xi_{n}\right]<\zeta<\xi^{*}$. Since the $a_{\xi}$ decrease, $a_{\zeta}<a_{\xi_{n}}$ for all $n$. Hence $n a_{5}<2^{n} a_{5}<2^{n} a_{\xi_{n}}<a_{1}$ for all $n$. Hence $a_{\zeta}, a_{1}$ are not relatively archimedean and the theorem is proved.

THEOREM 12. $X \in \bar{G}$ is non-Dedekindean if and only if there is a $y \neq \theta$ in $G$ such that $X+y=X$.

Proof. Suppose $X+y=X$ for $y \neq \theta$, then $X=X-y$ and either all of $X, X \pm y$ are Dedekindean or none is. Since $x \in X$ if and only if $x \pm y \in X \pm y$ the result follows from the definition of the Dedekindean property of lower segments.

TheOREм 13. If $X$ is non-Dedekindean there is no $Y \in \bar{G}$ such that $X+Y=\Theta$.

Proof. Since $X$ is non-Dedekindean, there is a $y \neq \theta$ such that $X+y=X$. Now we have the contradiction $X(y) \neq \Theta$ and if $X+Y=\Theta$,

$$
\Theta=X+Y=(X+X(y))+Y=(X+Y)+X(y)=X(y) .
$$

5. Hahn groups. The group $H$ given in the example of $\$ 3$ is a special Hahn group [5]. In general a Hahn group $H$ is the group of all real functions $x(\alpha)$ defined on an ordered set $A=[\alpha]$ such that the set $L(x)=[\alpha \mid x(\alpha) \neq 0]$ $C A$ is well-ordered in $A$. Order in $H$ is defined thus: $x>0$ if $x\left(\alpha_{0}\right)>0$ where $\alpha_{0}$ is the first element in $L(x)$ and $x_{1}>x_{2}$ if $x_{1}-x_{2}>\theta$. The Hahn groups are ordered abelian groups. Hence for each such group $H$ there is a minimal ordinal $\xi^{*}=\xi^{*}(H)$ and a decreasing sequence $x_{\xi} \in H$ such that $\xi^{*}$-lim $x_{\xi}=\theta$. Let us consider the initial elements $\alpha_{\xi}=L\left(x_{\xi}\right)$. Since the $x_{\xi}$ decrease the $\alpha_{\xi}$ do not decrease. If the set $\left[\alpha_{\xi} \mid \xi<\xi^{*}\right]$ has a maximum $\alpha_{0}$, then $a_{n}=(1 / n) x_{\alpha_{0}}$ is cofinal with $x_{\xi}$ in $H$ and $\xi^{*}=\omega$. If the set $\left[\alpha_{\xi} \mid \xi<\xi^{*}\right]$ has no maximum there is an increasing subsequence $\alpha_{\xi_{\eta}}, \eta<\xi^{*}$, cofinal with $\alpha_{\xi}$ in $A$ and the elements $a_{\eta} \in H$ such that 


$$
a_{\eta}(\alpha)= \begin{cases}0, & \alpha \neq \alpha_{\xi_{\eta}}, \\ 1, & \alpha=\alpha_{\xi_{\eta}},\end{cases}
$$

form a sequence decreasing to $\theta$ in $H$. For: To each $\alpha_{\xi}$ there is an $\alpha_{\xi_{\eta}}>\alpha_{\xi}$ and so $a_{\eta}<x_{\xi}$ while, since $\alpha_{\xi_{\eta}}$ increases in $A, a_{\eta}$ decreases in $H$. The neighborhoods $U_{\xi}(x)$ are defined in $H$ by:

$$
\begin{aligned}
U_{\xi} & =U_{\xi}(\theta)=\left[x|| x \mid<a_{\xi}\right], \\
U_{\xi}(x) & =x+U_{\xi},
\end{aligned}
$$$$
\xi<\xi^{*}
$$

An equivalent set of neighborhoods $V_{\alpha}(x)$ are defined by:

$$
\begin{aligned}
T_{\alpha} & =[\beta \mid \alpha \leqq \beta] \subset A, & \alpha \in A, \\
V_{\alpha} & =\left[x \mid L(x) \subset T_{\alpha}\right], & \\
V_{\alpha}(x) & =x+V_{\alpha}=\left[x+y \mid y \in V_{\alpha}\right] . &
\end{aligned}
$$

Since the groups $H$ are groups $G$, the neighborhoods have the properties of Theorem 3.

THEOREM 14. If $\eta^{*}<\xi^{*}(H)$ and $U_{\xi_{\eta}}\left(x_{\eta}\right)$ decrease on $\eta<\eta^{*}$, then $\bigcap_{\eta<\eta^{*}} U_{\xi_{\eta}}\left(x_{\eta}\right)$ is a nonempty open set.

Since $\eta_{1}<\eta_{2}<\eta^{*}$ implies $U_{\xi_{\eta_{1}}}\left(x_{\eta_{1}}\right) \supset U_{\xi_{\eta_{2}}}\left(x_{\eta_{2}}\right), \quad x_{\eta_{2}}-x_{\eta_{1}} \in U_{\xi_{\eta_{1}}}$ and $x_{\eta_{1}}(\alpha)$ $=x_{\eta_{2}}(\alpha)$ for $\alpha<\alpha_{\eta_{1}}$. Hence there is $x \in H$ such that $x(\alpha)=x_{\eta_{\eta}}(\alpha)$ for $\alpha<\xi_{\eta_{\eta}}$, $\eta<\eta^{*}$. Clearly $x \in \bigcap_{\eta<\eta^{*}} U_{\xi_{\eta}}\left(x_{\eta}\right)$ and the intersection is not empty. Finally if $x \in \bigcap_{\eta<\eta^{*}} U_{\xi_{\eta}}\left(x_{\eta}\right)$, then there is $U_{\xi_{\eta}}(x) \subset U_{\xi_{\eta}}\left(x_{\eta}\right)$ for $\eta<\eta^{*}$. Since sup $\left[\zeta_{\eta} \mid \eta<\eta^{*}\right]$ $=\zeta<\xi^{*}$ (Theorem 2), $U_{\zeta}(x) \subset \bigcap_{\eta<\eta^{*}} U_{\xi_{\eta}}(x) \subset \bigcap_{\eta<\eta^{*}} U_{\xi_{\eta}}\left(x_{\eta}\right)$ and the intersection is open.

This theorem shows that the Hahn groups satisfy axiom 4 of [1] and so all the axioms given there, since $\alpha, \beta, \gamma$ of Theorem 3 are 1, 2, 3 of [1]. Certain properties of Hahn groups are immediate.

Corollary 1. A Hahn group is t-complete.

Proof. Hahn showed in [5] that groups $H$ are $a$-complete. It follows from Theorem 9 that they are $t$-complete.

\section{Corollary 2. Hahn groups are of the second $\xi^{*}$-category.}

Proof. The Hahn groups satisfy axioms 1, 2, 3, 4 of [1] and it is shown there that $t$-complete (called $\xi^{*}$-complete) spaces are of the second $\xi^{*}$-category.

THEOREM 15. A lower segment $X$ of a Hahn group $H$ has a least upper bound if and only if it is Dedekindean $\left.{ }^{4}\right)$.

(4) A different proof of this result was communicated to us by D. Zelinsky. 
Proof. If sup $X=x_{0} \in H$, then $x_{0}-a_{\xi}$ is a fundamental sequence cofinal with $X$ such that $\left(x_{0}-a_{\xi}\right)+a_{\xi}=x_{0} \notin X$. By Theorem $5, X$ is Dedekindean. Now suppose $X$ is Dedekindean. Then by Theorem 5 there is a fundamental sequence $x_{\xi}$ cofinal with $X$ such that $x_{\xi}+a_{\xi} \notin X$. Since $H$ is $t$-complete, $\lim x_{\xi}=x_{0} \in H$ and for $\eta<\xi^{*}$ there is a $\xi_{\eta}>\eta$ such that for $\xi_{\eta} \leqq \xi<\xi^{*}, x_{0}-a_{\eta}$ $<x_{\xi}<x_{0}+a_{\eta}$. Now $x_{0} \in X$. Otherwise there is in $X$ a $y>x_{0}$. There is, by Lemma 1 , an $\eta<\xi^{*}$ such that $2 a_{\eta}<y-x_{0}$. Then for $\xi_{\eta} \leqq \xi<\xi^{*}, x_{\xi}+a_{\xi}<x_{0}$ $+a_{\eta}+a_{\xi}<x_{0}+2 a_{\eta}<y \in X$ and $x_{\xi}+a_{\xi} \in X$, which is false. Therefore, $x \in X$ implies $x<x_{0}$ which with $x_{0}-a_{\eta}<x_{\xi} \in X$ for all $\eta$ and some $\xi$ less than $\xi^{*}$ yields $x_{0}=\sup X$.

\section{BIBLIOGRAPHY}

1. R. Baer, Zur Topologie der Gruppen, J. Reine Angew. Math. vol. 160 (1929) pp. $208-226$.

2. L. W. Cohen and C. Goffman, $A$ theory of transfinite convergence, Trans. Amer. Math. Soc. vol. 66 (1949) pp. 65-74.

3. C. J. Everett, and S. Ulam, On ordered groups, Trans. Amer. Math. Soc. vol. 57 (1945) pp. 208-216.

4. D. Hilbert, Grundlagen der Geometrie, Leipzig, 1903.

5. H. Hahn Über die nichtarchimedischen Grössensysteme, Sitzungsberichte der Kaiserlichen Akademie der Wissenschaften, Vienna, Section IIa, vol. 116 (1907) pp. 601-653.

Queens College,

Flushing, N. Y.

UNIVERSITY OF OKLAHOMA,

NORMAN, OKLA. 